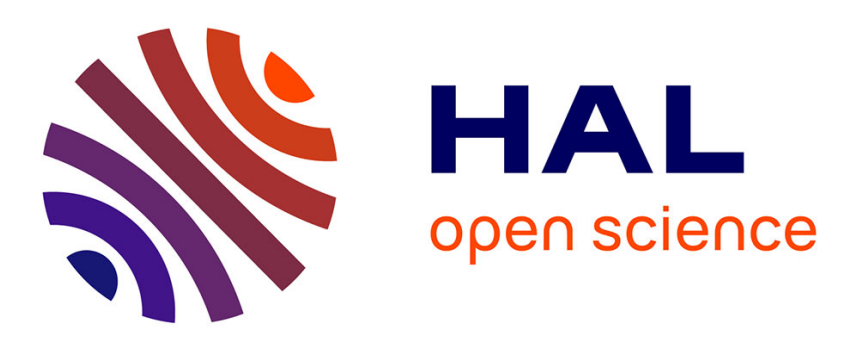

\title{
Convection Induced Self-Organization in Electroless Deposition Experiments
}

\author{
F. Texier, G. Gadret, Christophe Léger, Françoise Argoul
}

\section{To cite this version:}

F. Texier, G. Gadret, Christophe Léger, Françoise Argoul. Convection Induced Self-Organization in Electroless Deposition Experiments. Journal de Physique II, 1997, 7 (4), pp.663-675. 10.1051/jp2:1997150 . jpa-00248470

\section{HAL Id: jpa-00248470 https://hal.science/jpa-00248470}

Submitted on 1 Jan 1997

HAL is a multi-disciplinary open access archive for the deposit and dissemination of scientific research documents, whether they are published or not. The documents may come from teaching and research institutions in France or abroad, or from public or private research centers.
L'archive ouverte pluridisciplinaire $\mathbf{H A L}$, est destinée au dépôt et à la diffusion de documents scientifiques de niveau recherche, publiés ou non, émanant des établissements d'enseignement et de recherche français ou étrangers, des laboratoires publics ou privés. 


\title{
Convection Induced Self-Organization in Electroless Deposition Experiments
}

\author{
F. Texier, G. Gadret, C. Léger and F. Argoul (*) \\ Centre de Recherche Paul Pascal, CNRS, avenue A. Schweitzer, 33600 Pessac, France
}

(Received 12 September 1996, revised 22 November 1996, accepted 9 January 1997)

PACS.81.15.Pq - Electrodeposition, electroplating

PACS.47.20.Bp - Buoyancy-driven instability

PACS.47.54. $+\mathrm{r}$ - Pattern selection

\begin{abstract}
We present the first evidence for spatio-temporal merging dynamics in thin gap electroless deposition experiments. The influence of both the gap thickness and the buoyancy forces on these phenomena is analyzed. The occurrence of this reverse cascading dynamical process is discussed in terms of the interaction of two counter rotating buoyancy driven convection rolls.
\end{abstract}

\section{Introduction}

Metal displacement reactions (also named electroless deposition in a more general acceptance) have been recognized for their industrial importance in the recovery of metals and the purification of process streams since the nineteenth century [1]. If the general idea of metal displacement reactions, i.e. the possibility of depositing a more noble metal (less oxidizable) onto a less noble (more oxidizable) metal, has been widely exploited in industrial applications, the characterization of the local kinetics of these complex processes has remained for years far beyond the scope of the experimental techniques. Despite the fact that the transport processes have been early recognized as the major kinetics limiting steps [1-7], the interfacial reaction distribution and the spatio-temporal dynamics of an electroless reaction remain challenging questions. In this paper, we want to illustrate the implication of buoyancy forces in electroless deposition. In particular we select a particular experimental situation where we show that this buoyancy driven convection can also induce a very uncommon spatio-temporal organization which is described in Figure 1 by a set of successive snapshots of the growth process. This dynamical process, which is reflected by a spatial cascade of branch merging, has been obtained by the displacement of copper ions by zinc metal in a thin gap cell ( $250 \mu \mathrm{m}$ depth).

\section{Experimental Set-Up}

The electrolytic cell is made of two optically flat $(\lambda / 4)$ glass plates, sealed at a fixed distance $\delta$ with a hot glue. The gap between these plates is fixed by the size of two zinc wires which serve both as spacers and electrodes. This experimental device has already been described for

$\left(^{*}\right)$ Author for correspondence (e-mail: argoul@crpp.u-bordeaux.fr)

(C) Les Éditions de Physique 1997 

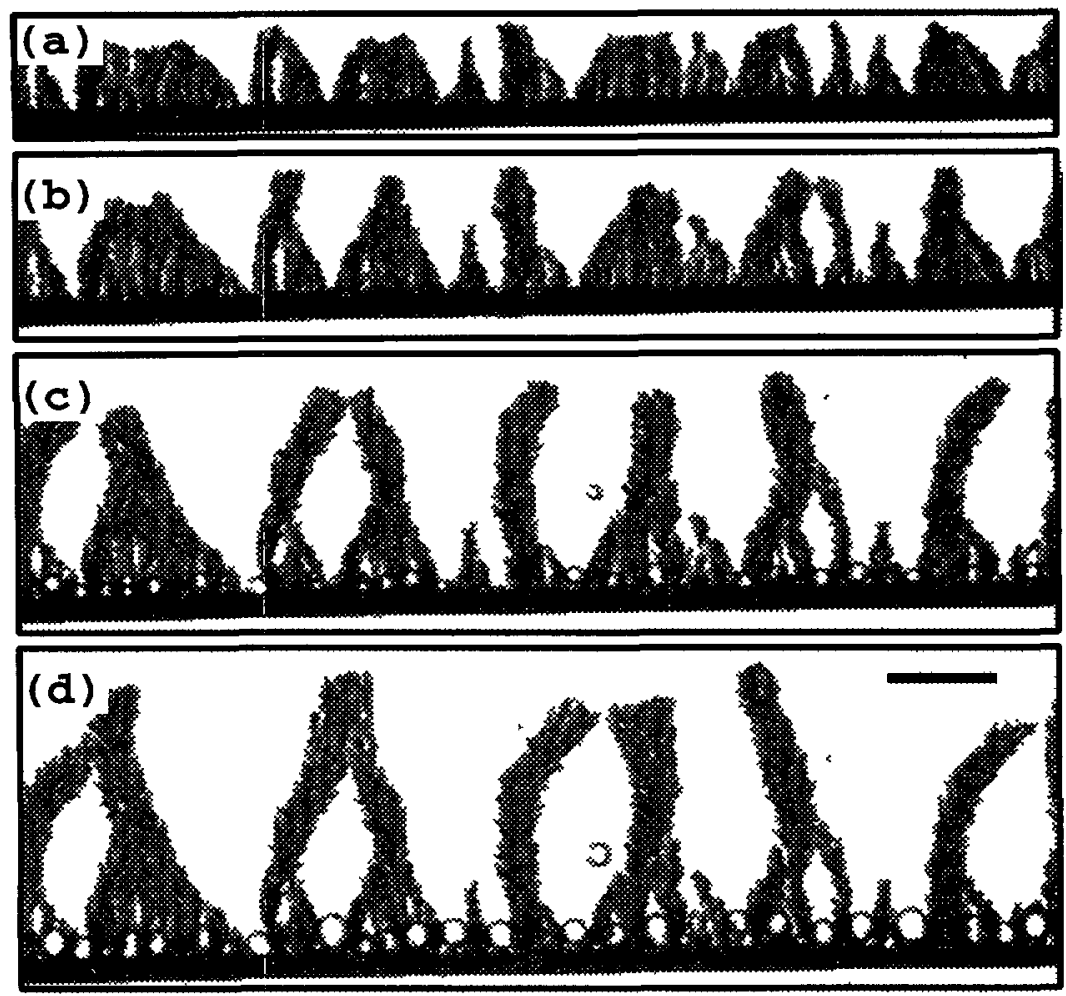

Fig. 1. - Direct visualization of the merging process in the cementation of copper ions on zinc. The four panels correspond respectively to the successive times: (a) $t=240 \mathrm{~s}$, (b) $420 \mathrm{~s}$. (c) $1020 \mathrm{~s}$, and (d) $1620 \mathrm{~s}$. Experimental parameters: $\left[\mathrm{CuSO}_{4}\right]=0.5 \mathrm{~mol} \mathrm{l}^{-1}$, width of the cell: $50 \mathrm{~mm}$, gap between the glass plates: $0.25 \mathrm{~mm}$, the black bar corresponds to $1 \mathrm{~mm}$.

electrodeposition of metals in reference [8]. The zinc wires (>99.9\%) of different thicknesses are purchased from Goodfellow and the copper sulfate electrolyte is prepared from a salt of purity 99.9\% (ACS reagent Aldrich). Nitrogen gas is also bubbled through the solution for at least one hour prior to use. The filling of the interspace delimited by the glass plates and the zinc wires is done by capillarity and it initiates immediatly a local oxido-reduction process without any external electronic circuit. The pictures of the growth are observed through microscope lenses and recorded on a video tape by a VCR. On a different set-up the concentration gradients inside the electrolyte can also be characterized by the measurement of its index by interferometry. The interferometric fringe patterns are obtained by a Mach Zehnder two-beam interferometer whose description can be found in reference [9].

\section{The General Features of Electroless Deposition}

Electroless deposition is a "null current" growth process since the oxidation of one species (here the zinc metal) must exactly compensate the reduction of some electrolyte ions, respectively copper and protons in the present study. Very schematically, one can write the global charge transfer mechanisms which are likely to occur on the zinc interface when it comes into contact 

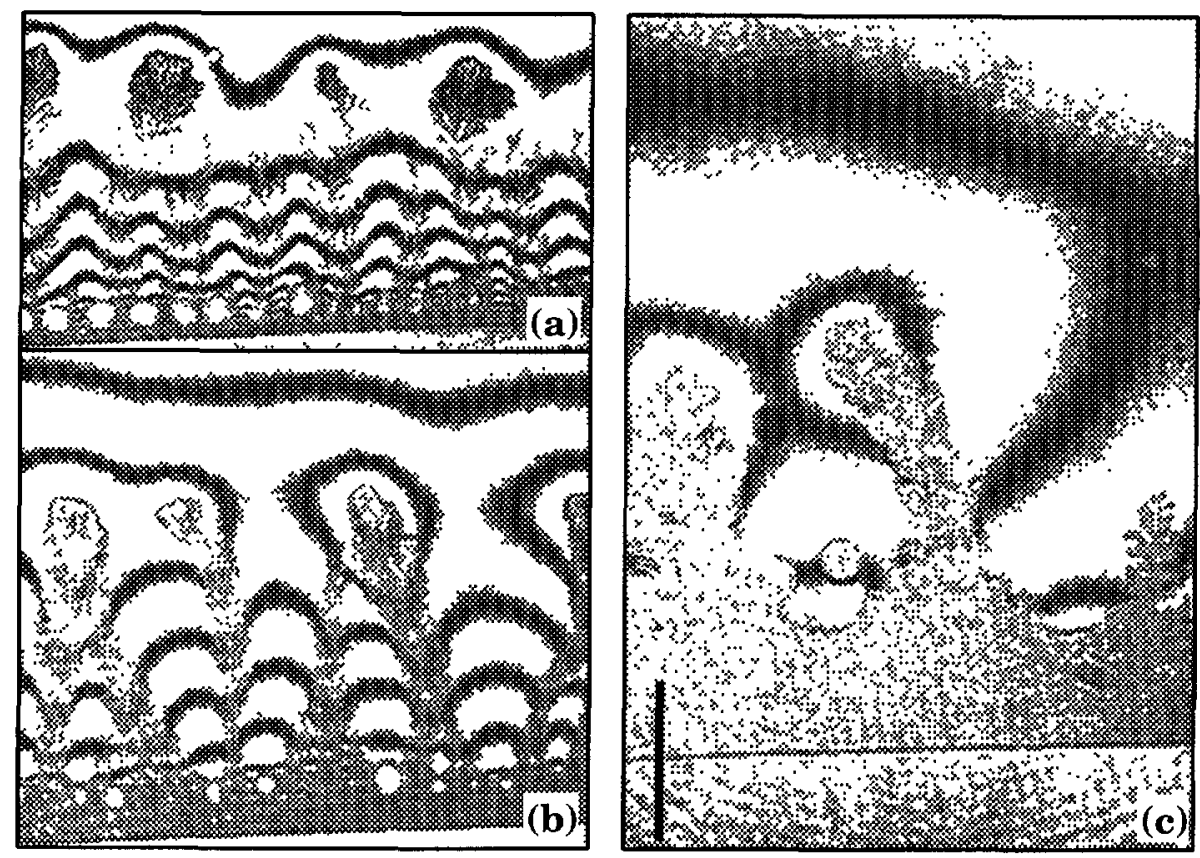

Fig. 2. - Interferometric visualization of the whole zone of index variation through the electroless deposition cell. The laser wavelength is $632.8 \mathrm{~nm}$ ( $\mathrm{He}-\mathrm{Ne}$ ). (a) cell gap $125 \mu \mathrm{m}$, (b) $250 \mu \mathrm{m}$, (c) $500 \mu \mathrm{m}$. The experimental parameters: $\left[\mathrm{CuSO}_{4}\right]=0.5 \mathrm{~mol} \mathrm{1}^{-1}$, width of the cell: $50 \mathrm{~mm}$, the black bar corresponds to $1 \mathrm{~mm}, t=25 \mathrm{~min}$ for the three pictures.

with the copper sulfate solution:

$$
\begin{array}{cccc}
\mathrm{Zn} & \longrightarrow & \mathrm{Zn}^{2+}+2 \mathrm{e}^{-} & \text {oxidation } \\
\mathrm{Cu}^{2+}+2 \mathrm{e}^{-} & \longrightarrow & \mathrm{Cu} & \text { reduction } \\
2 \mathrm{H}_{2} \mathrm{O}+2 \mathrm{e}^{-} & \longrightarrow & \mathrm{H}_{2}+2 \mathrm{OH}^{-} & \text {reduction . }
\end{array}
$$

We will make a priori the assumption that the oxidation of zinc and the reduction of copper can both be schematized by fast charge transfer processes and therefore that the dynamics of the growth will rather be limited by the transport processes. Our experimental findings will prove afterwards that this is the case. From the set of equations (1), we conclude that two opposite concentration gradients, respectively due to the dissolution of zinc ions (increasing concentration close to the zinc wire) and the reduction of copper ions (depletion of the neighborhood of the copper deposit) will rapidly set in after filling the cell.

By interferometric analysis we get a real time visualization of the index of the electrolyte, as shown in Figure 2. Each fringe corresponds to a line of constant phase or constant index, averaged over the depth of the cell. In electroless deposition, since the electrolyte is made of two different salts (copper and zinc sulfate) the interpretation of these patterns is not as straightforward as in the case of electrodeposition [9]. Although our analysis will not be completely quantitative here, we want to show through the qualitative examination of the three panels in Figure 2 that we can already get some hints to interpret the merging process. We observe in Figures $2 a$ to $2 c$ the separation of two zones along the growth. The first zone is the nearest to the zinc wire and is represented in Figure 2a by very closely spaced fringes (constant phase fringes) which brings evidence that the concentration of zinc sulfate is quickly 

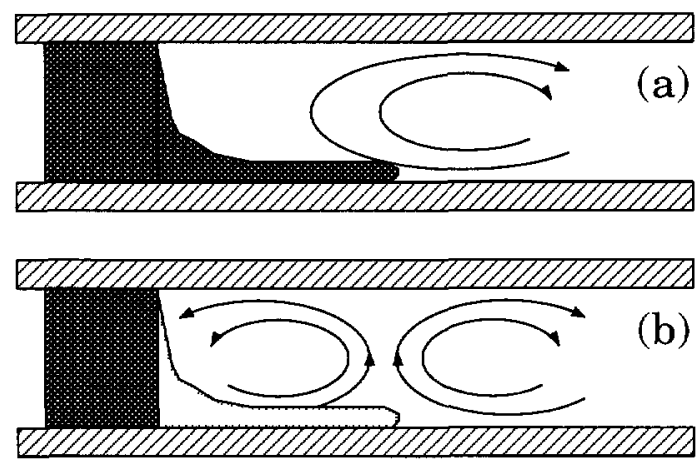

Fig. 3. - Buoyancy driven convection in electrodeposition processes. (a) Sketch of the convective roll in the case of a fixed current electrodeposition experiment. (b) Two counter rotating convective rolls are observed in electroless deposition experiment. As sketched in these pictures in a rather naive way, the deposit does not fill the whole interspace between the glass plates; it actually occupies a finite fraction of this gap which depends on the gap size and the other experimental parameters. Let us note the different shaded patterns which have been chosen to symbolize the zinc (black background with white dots) and the copper (white background with black dots).

increasing (repletion of zinc cations) when one gets closer to the zinc electrode. The second zone is located in the neighborhood of the tips of the copper trees and is made of more loosely spaced fringes. This second zone must be related in part to the depletion of copper sulfate by the reduction of copper ions. In between these two zones, the two concentration gradients mix together. If the transport were purely diffusive, one would expect the number of fringes in front, as well as in the rear of the growth to increase linearly with the gap of the cell. This is obviously not the case in Figure 2 since this number of fringes saturates in front of the growing zone when the gap of the cell is increased. Furthermore, the number of fringes close to the zinc wire drops to zero for $500 \mu \mathrm{m}$ gap cells. This is an unambiguous evidence for the enhancement of the mixing of the two zones of concentration gradient with the size of the gap of the cell. We discuss the nature of this mixing in terms of buovancy driven convection in the next paragraph.

\section{Buoyancy Driven Convective Instabilities in Electroless Deposition}

Buoyancy-driven convection has been very early invoked in the electrochemical literature as an important ingredient of interfacial transport process $[6,7,10-15]$. We have analyzed in reference [10] the emergence of buoyancy driven convection in thin gap electrodeposition experiments, under galvanostatic control. The creation of a concentration depletion zone in front of a metal cathode triggers a convective roll (such as sketched in Fig. 3a) which spreads across the cell with a square root law of time. This convection is the predominant transport process for cell gaps greater than $125 \mu \mathrm{m}$. We can extract from the plots of reference [10] the logarithmic dependence of the maximum vertical fluid velocity versus the gap $\delta: V \propto \log (\delta)$. We have represented in Figure 4 the evolution of the internal velocity of the buoyancy driven convective roll for electrodeposition experiments and we show that this velocity can be extrapolated to zero when the gap of the cell approaches $50 \mu \mathrm{m}$. These measures have been obtained for fixed current experiments and confirmed by a theoretical analysis of Chalzaviel and collaborators in reference [7]. We think moreover that the influence of the depth of the cell should be quite the same for electroless deposition. 

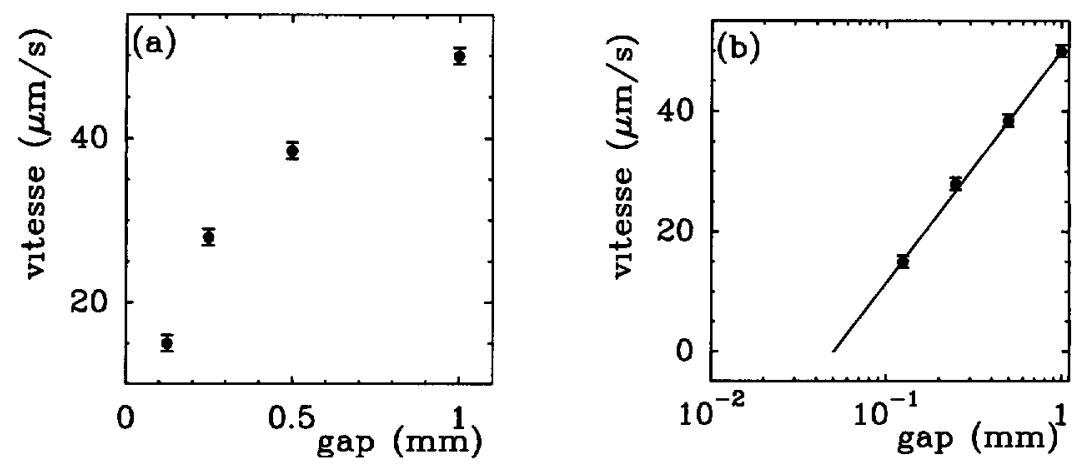

Fig. 4. - Dependence of the buoyancy driven maximum velocitv versus the size of the gap of the cell. (a) lin-lin plot, (b) lin-log plot of $V$ versus the gap size $\delta$, the mean square fit of these data brings the prediction of the damping of this convection around $50 \mu \mathrm{m}$ gap size. Experimental parameters: $\left[\mathrm{ZnSO}_{4}\right]=0.1 \mathrm{~mol} \mathrm{l}^{-1}, J=40 \mathrm{~mA} \mathrm{~cm}{ }^{-2}, t=500 \mathrm{~s}$, width of the cell: $50 \mathrm{~mm}$, length of the cell: $100 \mathrm{~mm}$.

In electroless deposition, the system involves two opposite concentration gradients which are going to trigger contra-rotating convection rolls such as sketched in Figure $3 \mathrm{~b}$. At the beginning of the electroless deposition experiment, the two concentration gradients are confined in a narrow zone in front of the zinc wire, the depletion of the copper ions is very fast and the deposition of copper starts quasi-instantaneously after filling the cell. On the contrary to the galvanostatic electrodeposition, there is no induction period and the flat interface of zinc wire is immediatly unstable. This immediate separation of the zone of dissolution of zinc ions and the front of depletion of copper ions builds on separate zones with opposite concentrations gradients which will be the field for two contra-rotating buoyancy driven convective rolls. This phenomenon is rather difficult to analyze by a direct inspection of the local velocity of the fluid because it is a global process which rapidly involves the whole set of growing branches. Moreover, this process is intrinsically non-stationary. Through the evidence of the spreading and rarefaction of interference fringes when the gap of the cell is increased (Figs. 2a to 2c). the interferometric analysis brings the clue that buoyancy driven convection supersedes diffusion from the early beginning of the growth.

\section{Experimental Demonstration of the Influence of Gravity}

To provide a definite demonstration of the convective origin of the merging process, we propose two sets of experiments. The first one is based on the modification of the cell gap. The second one uses an experimental trick to modify the force of gravity perpendicular to the glass plates of the cell.

In Figure 5, we show the evolution of the morphology of copper electroless deposits for cell gap ranging from $50 \mu \mathrm{m}$ to $500 \mu \mathrm{m}$. For very small gap cells $(50 \mu \mathrm{m}$, Fig. $5 \mathrm{a})$, there is a strong perturbation of the process by the gas release $\left(\mathrm{H}_{2}\right)$ from the proton reduction which leads to bubble formation. The morphology is very reminiscent of those which have been previously discussed in terms of diffusion-limited aggregates [4]. From $125 \mu \mathrm{m}$ to $250 \mu \mathrm{m}$ gap, there is a drastic modification of the morphology (Figs. 5b and 5c); the deposit is much denser at the beginning and moreover one observes the self-organization of the deposit through a cascade of merging between adjacent branches. Above $250 \mu \mathrm{m}$, if we choose a $500 \mu \mathrm{m}$ gap cell as 

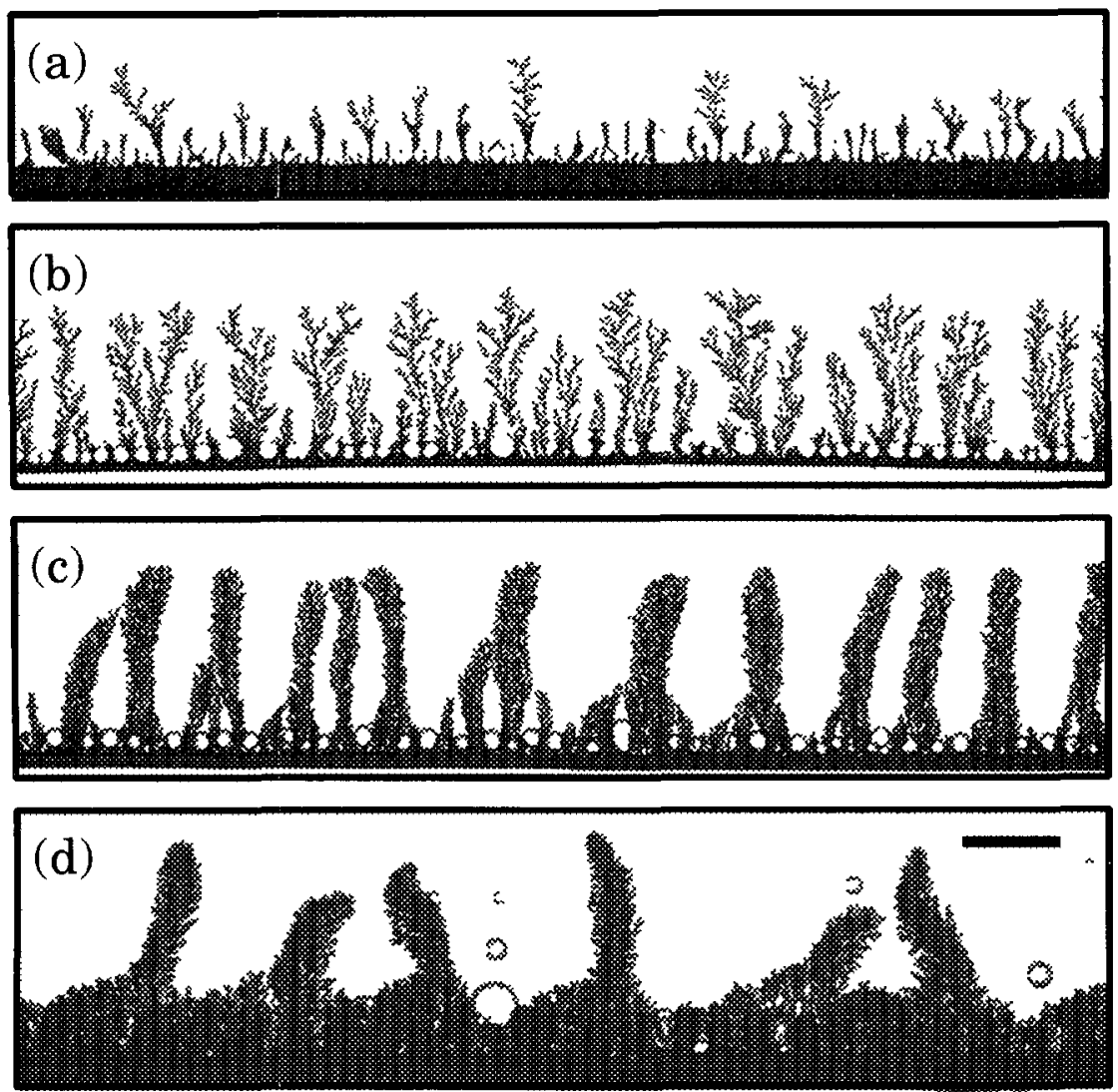

Fig. 5. - Electroless deposition in different gap size cells. The fours panels correspond respectively to the gaps: (a) $d=50 \mu \mathrm{m}$, (b) $125 \mu \mathrm{m}$, (c) $250 \mu \mathrm{m}$ and (d) $500 \mu \mathrm{m}$. Experimental parameters: $\left[\mathrm{CuSO}_{4}\right]=0.5 \mathrm{~mol} \mathrm{l}^{-1}$, width of the cell: $50 \mathrm{~mm}$, the black bar corresponds to $1 \mathrm{~mm}, t=20 \mathrm{~min}$ for the four pictures.

in Figure 5d, we still observe the emergence of narrow fingers from an initial dense and flat deposit, followed by the progressive merging of these dense fingers. When further increasing the gap of the cell (beyond $500 \mu \mathrm{m}$ gap), the mixing of the fluid by convection is too strong to give a definite structuration of the branches.

In Figure 6, is reported a set of experiments with cells that have been tilted with respect to their initial horizontal position. If we increase the tilting angle a between the horizontal axis and its projection onto the plates of the cell, we increase the component of the gravity force which is parallel to the direction of the growth and therefore we decrease its component which is perpendicular to the glass plates. In Figure 6, both sides of the central zinc electrode have been filled by a copper sulfate solution and the orientation of the gravity $\mathbf{g}$ gives the direction of the component of this force which is parallel to the glass plates. The first picture of Figure 6a corresponds to a tilting angle of $15^{\circ}$. This picture shows the asymmetry of the electroless process when the electrolyte is respectively above or below the zinc wire where the growth starts. We observe that when the wire is below the electrolyte, this leads to an immediate disappearance of the merging process; this is probably due to the stabilization of the convection originating from zinc dissolution by sedimentation of the zones of higher 

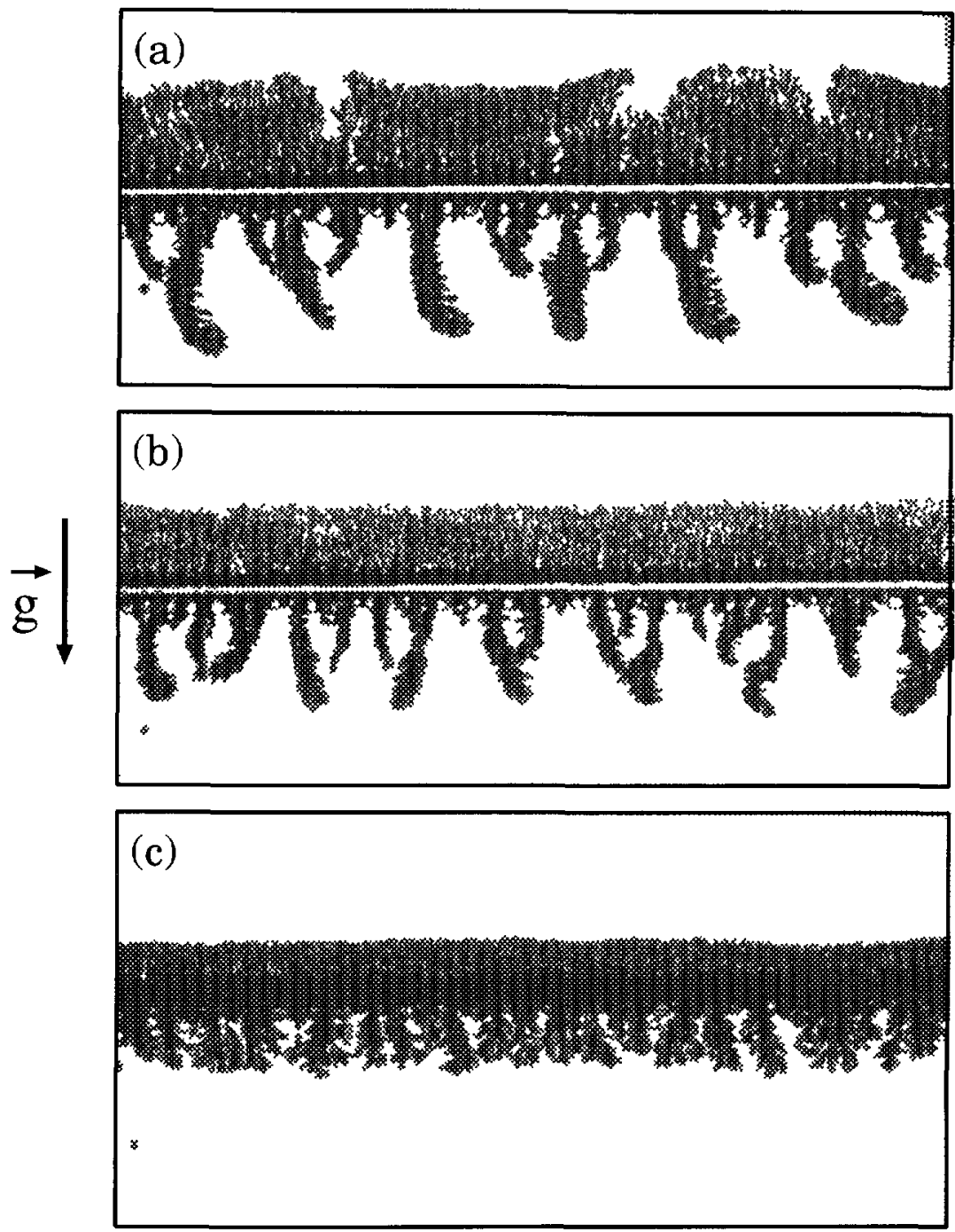

Fig. 6. - Influence of the gravity force on the dynamics of electroless deposition. The cell is tilted and the zinc wire is surrounded on both sides by the copper sulfate electrolyte. The four pictures correspond respectively to a tilting angle: (a) $\alpha=15^{\circ}$, (b) $25^{\circ}$ and (c) $45^{\circ}$. Experimental parameters: $\left[\mathrm{CuSO}_{4}\right]=0.5 \mathrm{~mol} \mathrm{l}^{-1}$ width of the cell $50 \mathrm{~mm}, t=10 \mathrm{~min}$ for the three pictures, width of the pictures: $24 \mathrm{~mm}$.

concentration onto the zinc wire. Whereas the copper density gradient in front of the deposit leads to a destabilization of this zone by buoyancy forces because of its lighter density when the cell is tilted. This zone is more rapidly mixed and when the angle of tilting is further increased (Figs. 6b and 6c) there must even be some plumes of lighter fluid which invade the upper part of the cell. On the contrary, when the wire is over the electrolyte, the zinc density gradient builds a heavier density fluid which is going to fall down and possibly trigger a Rayleigh-Taylor [17] type instability which enhances the mixing of zinc ions towards the depletion layer in front of the deposit, while the copper density gradient remains confined in front of the growth. When $\alpha$ 
is increased, the merging process is first strengthened (Figs. 6a and 6b) because the buoyancy forces amplify the mixing of the two convective roll zones and then it disappears completely (Fig. 6c), leaving a branched growth pattern which is sparser than the dense pattern which is obtained when the zinc electrode is below the electrolyte.

\section{Interpretation of the Interplay of Buoyancy Convections}

Since the convection surely plays a crucial role in the initiation of the observed merging process, the modeling of the coupling of fluid dynamics with the interface deformation remains a fundamental challenge. Let us attempt to provide a preliminary interpretation of the main ingredients underlying this spatio-temporal process. We recall first the different stages of the electroless deposition. When the cell is filled with the copper sulfate solution, the initial flat zinc wire becomes instantaneously unstable because the concentration of copper ions drops to zero at the zinc interface. At the beginning of the experiment there is no buoyancy driven convection yet and the two zones of concentration gradient which are created by the oxidation and reduction processes are mixed as long as the edge of the copper deposit is not too far from the zinc wire. Indeed, as soon as the cell is filled, a stack of small copper branches rapidly emerges from the zinc wire and spreads apart the two concentration gradient zones respectively due to the copper ions reduction (depletion) and to the zinc metal oxidation (enrichment). These two zones of concentration gradients are likely to be the field for buoyancy driven convection, as guessed in our previous experimental evidences [10], for gap size greater than $125 \mu \mathrm{m}$. Moreover, the morphology and the progression velocity during this first regime of deposition depends on the gap of the cell and on the strength of the buoyancy driven convection triggered by the depletion of the copper sulfate solution. When the growth is dense (i.e. for cell gap size greater than $125 \mu \mathrm{m}$ ), the zinc replenishing zone, close to the zinc wire, is confined to a narrower gap size cell because the dense and bushy deposit falls towards to lower plate of the cell and fills a finite portion of the interval between the two glass plates. There is therefore, above the deposit, a superconcentration of zinc cations which make the fluid heavier. This denser fluid, by gravity tends naturally to fall down and to open, inside the dense branches of the deposit, free zones where the buoyancy driven convection can spread into localized convective rolls, bounded by dense fingers. This results in the breaking of the translational invariance of both the morphology of the deposit and the buoyancy driven convection, and indeed we observe the confinement of the growth into narrow dense fingers. Such phenomena could not be observed in a simple electrodeposition experiment where there is no buoyancy driven convection behind the tips of the growing branches. The emergence of these fingers modifies on its turn the "copper depletion' convection, in front of the fingers. The initially flat convective roll in front of the growth is disrupted into an array of small convective rolls, localized at the tips of the branches. In the rear of the branches, the "zinc repletion" convection is confined between adjacent branches and tends to widen them apart. The bending of the branches is not a simple mechanical effect due to convection but rather a progressive torsion of the branches towards the strongest concentration gradients which themselves depend on the shape and strength of convection and diffusion. The complexity in this dynamical process comes from the fact that the spatial organization of the branches modifies on its turn the transport by convection. If there were no "zinc repletion" convection, the growth would surely remain dense as long as the copper ions are fed in the cell. It is indeed what is evidenced in the upper side of the three panels $\mathrm{a}, \mathrm{b}$ and $\mathrm{c}$ of Figure 6 when the cell is tilted.

To bring a dynamical discussion of the recurring nature of this merging process, we have reconstructed space-time maps by extracting very thin slices from the interferometric video film of the growth. In Figures 7, 8 and 9, we show the reconstruction of these maps for different 


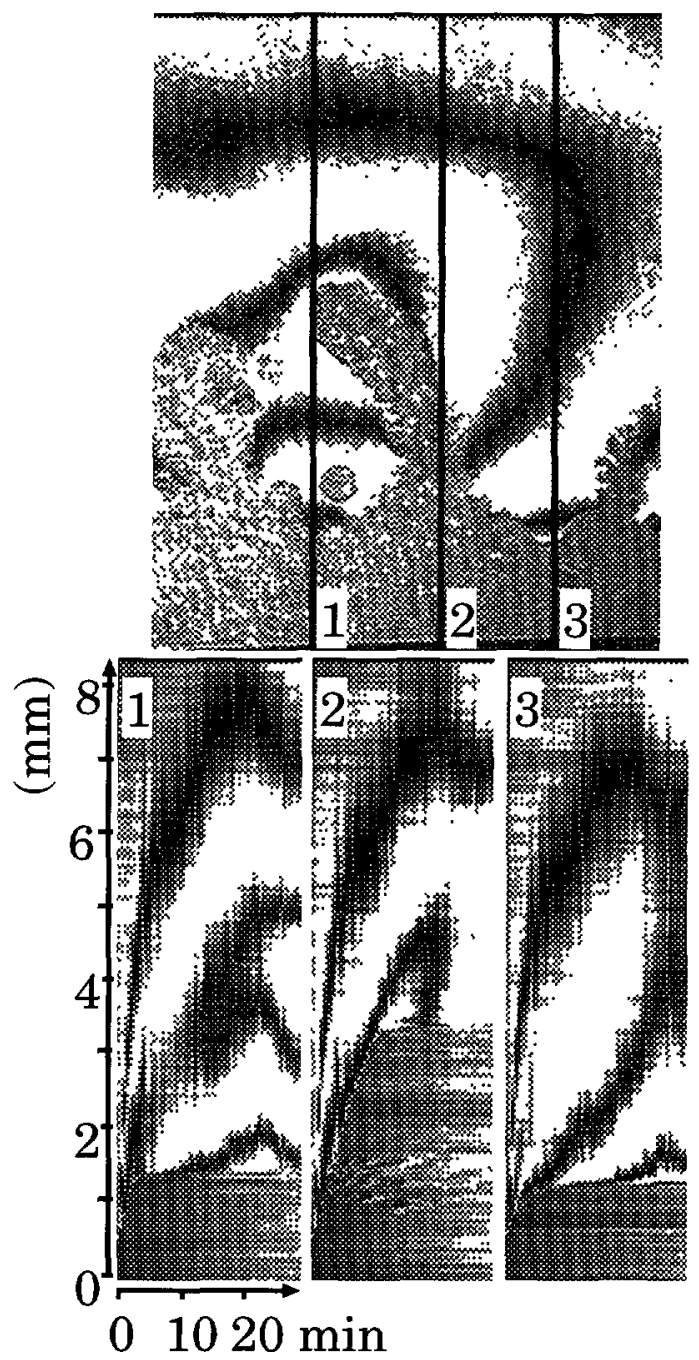

Fig. 7. - Space time reconstruction of the dynamics of electroless deposition in $500 \mu \mathrm{m}$ gap size cell. Each panel 1,2 and 3 has been reconstructed by the juxtaposition of 3 pixel wide slices selected from the original interferometric picture at fixed time interval $(30 \mathrm{~s})$. The selected slices are represented by black lines on the final interferometric picture of the growth $(t=30 \mathrm{~min}$ ) (above the three panels 1, 2 and 3). Experimental parameters: $\left[\mathrm{CuSO}_{4}\right]=0.5 \mathrm{~mol} \mathrm{l}^{-1}$, width of the cell $50 \mathrm{~mm}$.

gap size cells, respectively $500 \mu \mathrm{m}, 125 \mu \mathrm{m}$ and $250 \mu \mathrm{m}$. In this representation, the evolution of a very thin slice of the picture of the growth is plotted versus the time. The extracted slices are represented by black lines on the final picture of the growth which is reported on top of each figure. One can therefore straightforwardly visualize the time evolution (velocity) of each interference fringe and of the interface of the deposit. For each gap size we have selected three different slices at different positions of the cell, which we think to be representative of the dynamics of the growth process. In Figure 7, we show the $500 \mu \mathrm{m}$ gap cell; we observe a rery steep acceleration of the growth and of the fringe displacement, immediately followed by an abrupt slowing down and recession of the fringes. This phenomenon is totally unpredictable 


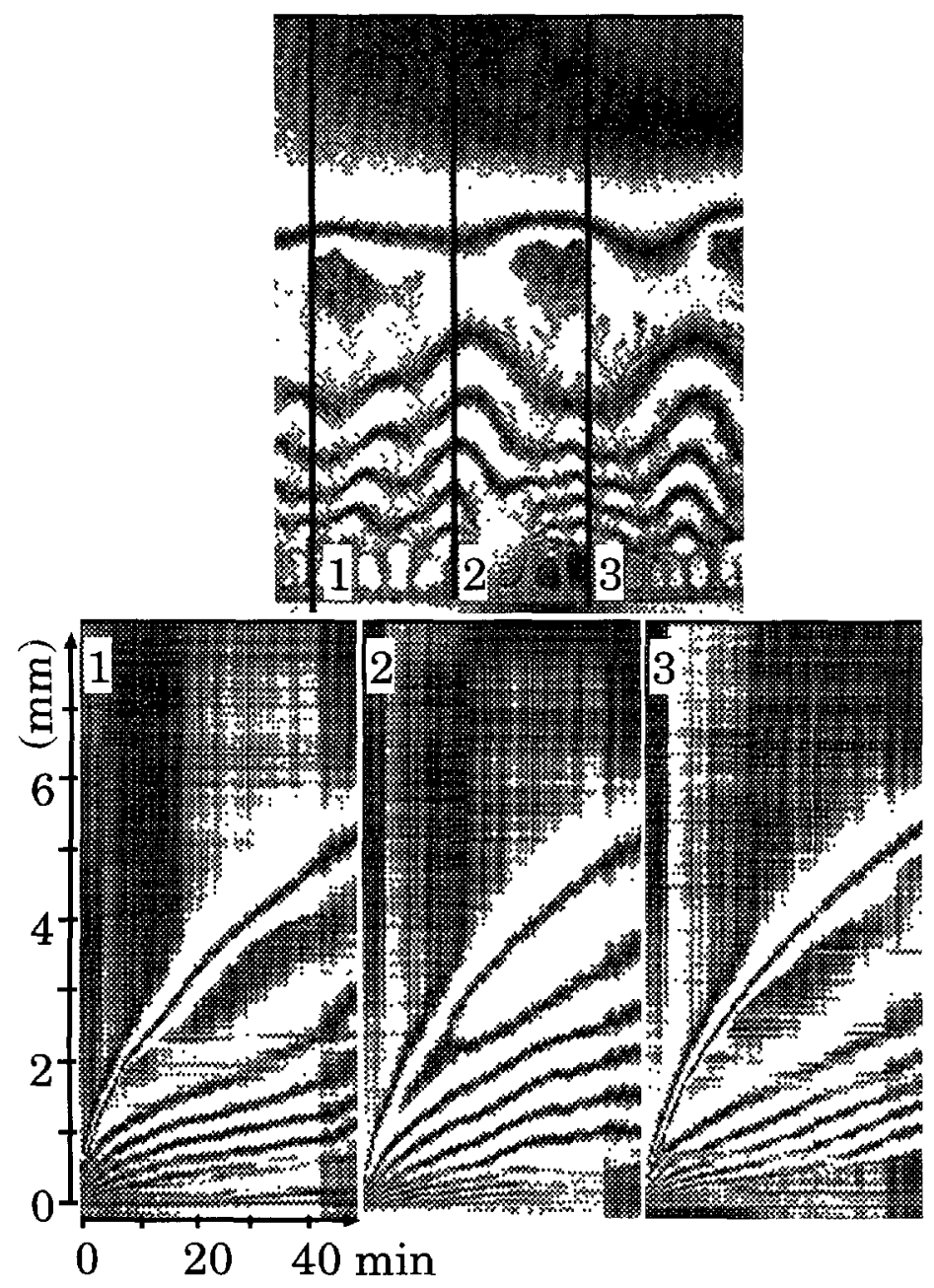

Fig. 8. - Space time reconstruction of the dynamics of electroless deposition in $125 \mu \mathrm{m}$ gap size cell. Each panel 1, 2 and 3 has been reconstructed by the juxtaposition of 3 pixel wide slices selected from the original interferometric picture at fixed time interval (30 s). The selected slices are represented by black lines on the final interferometric picture of the growth $(t=49 \mathrm{~min}$ ) (above the three panels 1,2 and 3). Experimental parameters: $\left[\mathrm{CuSO}_{4}\right]=0.5 \mathrm{~mol} \mathrm{l}^{-1}$, width of the cell $50 \mathrm{~mm}$.

if one does not invoke convection mechanisms for mixing thoroughly the cell. Figures 8 and 9 correspond respectively to gap sizes of $125 \mu \mathrm{m}$ and $250 \mu \mathrm{m}$. The first striking observation which comes from this dynamical representation is the fact that the propagation velocities of the fringes are twice bigger in the $250 \mu \mathrm{m}$ gap cell than in the $125 \mu \mathrm{m}$ gap cell. Moreover, in the finer gap cell $(125 \mu \mathrm{m}$, Fig. 8$)$, where the buoyancy driven convection is partly damped, the fringes behind the tip of the copper branches follow a quite regular and homogeneous (in space) dynamical evolution. In the $250 \mu \mathrm{m}$ gap cell (Fig. 9), the dynamics of these fringes is noticeably different, since for example in the panels 1 and 3 of Figure 9 , one observes a sudden slowing down of the fringes behind the growth around $10 \mathrm{~min}$. The slice of panel 1 corresponds to the heart of a merging zone. This slowing down of the fringes is likely to reflect 


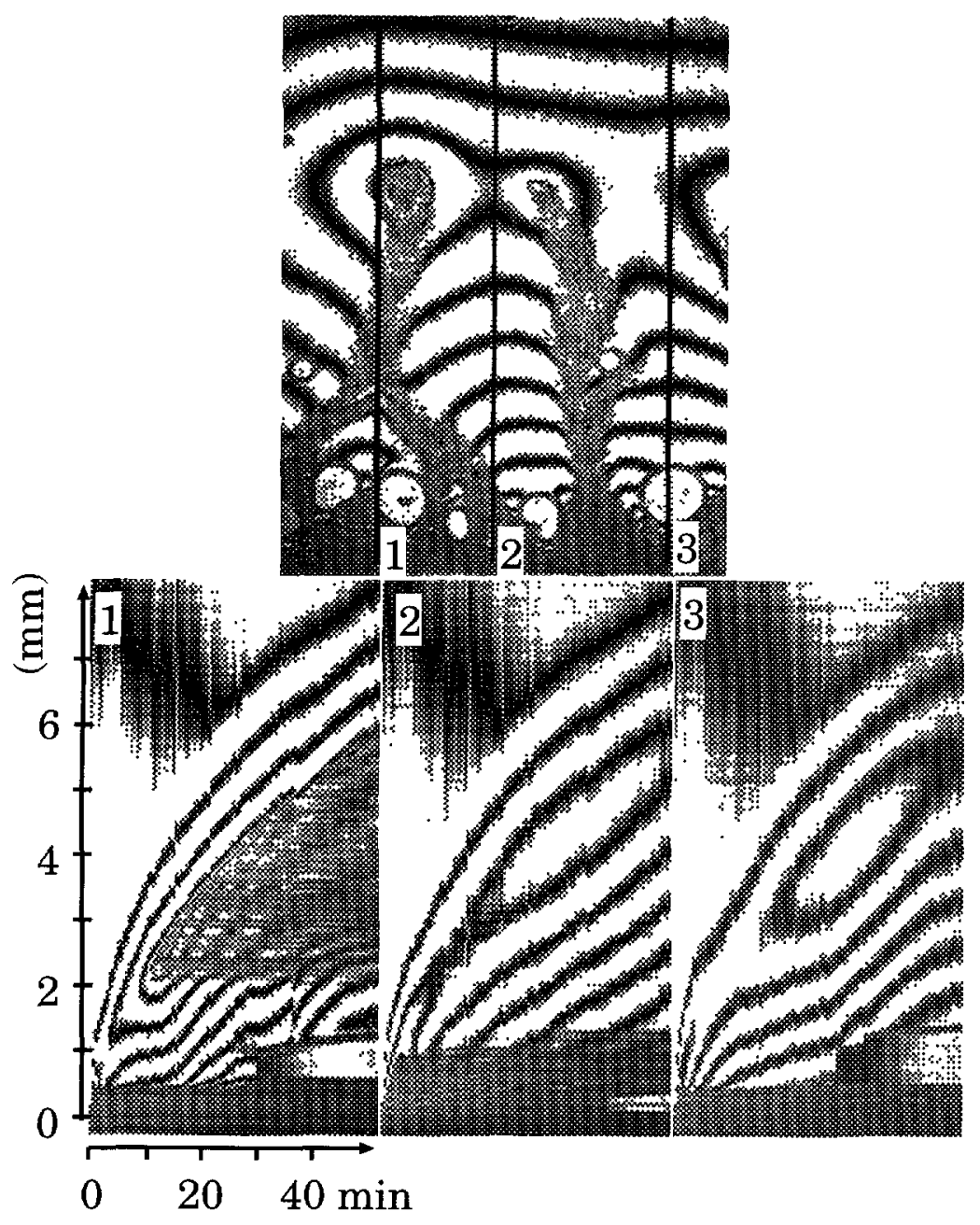

Fig. 9. - Space time reconstruction of the dynamics of electroless deposition in $250 \mu \mathrm{m}$ gap size cell. Each panel 1, 2 and 3 has been reconstructed by the juxtaposition of 3 pixel wide slices selected from the original interferometric picture at fixed time interval $(30 \mathrm{~s})$. The selected slices are represented by black lines on the final interferometric picture of the growth $(t=52 \mathrm{~min}$ ) (above the three panels 1, 2 and 3). Experimental parameters: $\left[\mathrm{CuSO}_{4}\right]=0.5 \mathrm{~mol} \mathrm{I}^{-1}$, width of the cell $50 \mathrm{~mm}$.

a local damping of the kinetics of the oxidation of zinc metal. When the two branches merge (around $12 \mathrm{~min}$.), these fringes undergo an acceleration in their dynamics, this phenomenon can reasonably be related to the confinement of this zone of electrolyte, behind the merged branches; since the "zinc" convection is therefore trapped inside a small part of the cell, it is likely to be amplified. This activation will be only temporary since the fringes will be slown down again, probably because the concentration of the zinc ions comes to saturation and the proton reduction gets more involved. In the panel 2 of Figure 9, we do not notice any slowing down in the progression of the fringes, as long as we choose a slice which is at equal distance from the neighboring branches. In the panel 3 of Figure 9, there is an inflexion of the fringes, which seems difficult to interpret since, as in the configuration of panel 2, the two neighboring 
branches have not yet merged. If we look more carefully at the global picture of the growth in Figure 9, we notice on the left branch, close to the slice 3, a side branch which is no longer growing at the time of this picture but which has probably perturbed earlier the dynamics of the zinc repletion zone. In this case there has been only a partial screening of the convection since the fringes underwent afterwards a renewed acceleration. The final evolution of this zone of fringes is strongly perturbed by the formation of $\mathrm{a}_{2}$ bubble which pushes the fluid ahead.

Finally we have also observed that the hydrodynamic sweeping of the head and the sides of the growing deposit by the zinc replenished fluid modifies the interfacial chemistry. In particular, during the growth. there is a local modification of the color of the deposit, which is black on the back of the branches and red at the tips. We have performed ex-situ Raman spectroscopy of the deposit which tends to prove that the deposit is probably made of a mixing of copper (0) and copper (I) with oxygen and that when the zinc ions are swept along the "copper" deposit by convection there might occur a codeposition of zinc (or zinc oxide) which passivates further the deposition of copper. These analysis are currently under progress and we should be able to perform these experiments in situ in a near future.

To conclude this discussion, we can stress again the fact that in Figure 9 the dynamics of the branches and the fringes shows a great variability in space and time when buoyancy forces are involved in the selection mechanism. This set of experiments brings also the clue that the kinetics of oxidation of zinc varies along the zinc metal interface, and that it is strongly coupled to transport processes, in this case buoyancy driven convection.

\section{Conclusion}

To conclude, we want to emphasize again the fundamental importance of buoyancy driven convection in the context of electrodeposition experiments. In the present work, we have seen that, besides some enhancement of the transport process, parallel to the growth through the cell, natural convection can trigger higher order non linear transport dynamics which is likely to affect the morphology of the copper deposit. We hope in a future communication to report on simultaneous measurements of each cation concentration profile to corroborate this demonstration. This work, which requires a substantial modification of our optical set-up, is currently under progress.

\section{Acknowledgments}

We are verv endebted to A. Arneodo for stimulating questions which improved the interpretation part of this paper and to A. Kuhn and to E. Galobardes for fruitful discussions. This work was supported by the Centre National d'Études Spatiales under Grant $n^{\circ} 96 / 0233$. One of us (G.G.) is very grateful to the Centre National d'Études Spatiales for its financial support under Grant n 95/0380.

\section{References}

[1] Power G.P. and Ritchie I.M., Metal Displacement Reactions, in "Modern Aspect of Electrochemistry", vol. 11 (1975) p. 199.

[2] Mogi I., Okubo S. and Nakagawa Y., J. Phys. Soc. Jpn 60 (1991) 3200.

[3] Paranjpe A.S., Bhakay-Tamhane S. and Vasan M.B., Phys. Lett. A 140 (1989) 193. 
[1] Kuhn A., Argoul F., Muzy J.F. and Arneodo A., Phys. Rev. Lett. 73 (1994) 2998.

[5] Kuhn A. and Argoul F., J. Electroanal. Chem. 397 (1995) 93.

[6] Barkey D., J. Electrochem. Soc. 141 (1994) 1206-1212.

[7] Chazalviel J.-N., Rosso M., Chassaing E. and Fleury V., J. Electroanal. Chem. 407 (1996) 61-73.

[8] Argoul F. and Kuhn A., J. Electroanal. Chem. 359 (1993) 81; Physica A 213 (1995) 209.

[9] Argoul F., Freysz E., Kuhn A., Léger C. and Potin L., Phys. Rev. E 53 (1996) 1777.

[10] Huth J.M., Swinney H.L., McCormick W.D., Kuhn A. and Argoul F., Phys. Rev. E 51 (1995) 3444.

[11] Agar J.N., Discussions Faraday Soc. 1 (1947) $26-37$.

[12] Wagner C., J. Electrochem. Soc. 95 (1949) 161-173.

[13] Tobias C.W., Eisenberg M. and Wilke C.R., J. Electrochem. Soc. 99 (1952) 359C-365C.

[14] Ibl N. and Müller R.H., J. Electrochem. Soc. 105 (1958) 346-353.

[15] Levich V.G., Physiochemical hydrodynamics (Prentice Hall, Englewood Cliffs, N.J., 1962).

[16] Witten T.A. and Sander L.M.. Phys. Rev. Lett. 47 (1981) 1440; Phys. Rev. B 27 (1983) 5686.

[17] Guyon E, Hulin J.P. and Petit L., Hydrodynamique physique (InterEditions, Éditions du CNRS, 1991). 